RISK FACTORS FOR PREVALENCE SYMPTOMS OF ECZEMA IN POLISH CHILDREN ACCORDING TO ECAP STUDY

\author{
A.J. Sybilski ${ }^{1,2}$, M. Michalczuk², M. Zalewska ${ }^{1}$, \\ B. Samoliński ${ }^{1}$ \\ ${ }^{1}$ Department of the Prevention of Environmental \\ Hazards and Allergology, Medical University \\ of Warsaw, ${ }^{2}$ Department of Paediatric and \\ Neonatology, Central Clinical Hospital of Ministry \\ of Internal Affairs, Warsaw, Poland
}

The aim of this paper was to identify familial and environmental factors that are associated with increased $A D$ risk in children.

Material and methods: Epidemiology of Allergic Diseases in Poland study (ECAP) was randomized, multicentre, cohort study, based on personal national identification number, performed in 2006-2008. The completed questionnaires were collected from 9231 children. According to ISAAC protocol 6-7 year-old $(n=4510)$ and $13-14$ year-old children $(n=4721)$ participated in ECAP study. The questionnaire based on ISAAC was used. Statistical significance was defined as $p<0.005$.

Results: Of the total 9231 children AD symptoms were observed in 3908 (42.3\%) (45.8\%;n=2066 and $39.0 \% ; n=1842)$. There was an increase risk of eczema among tobacco smokers $(35.0 \%)$ vs no smokers $(32.4 \%)(\mathrm{OR}=1.11,95 \% \mathrm{Cl}: 1.02-1.21)$. The eczema prevalence in household with visible moulds was $44.7 \%$ and without moulds $35.5 \%(O R=1.47$; 95\% Cl: 1.38-1.57). Multivariate logistic regression analysis showed that more frequent $A D$ symptoms correlated with older mother $(\mathrm{OR}=1.20 ; 95 \% \mathrm{Cl}$ : 0.96 1.49). Strong associations were found between the number of cars and lorries went near patient's home and more eczema presence (respectively: $\mathrm{OR}=1.64$, 95\% Cl: 1.22-1.75; OR=1.16, 95\%Cl: 1.06-1.26). High socioeconomic status and high prevalence of $A D$ were strong correlated $(\mathrm{OR}=1.37 ; 95 \% \mathrm{Cl}$ : 1.26 1.48). There was a significant association between spending more than 1 hour per day watching TV and less prevalence of eczema $(\mathrm{OR}=0.83 ; 95 \% \mathrm{Cl}$ : 0.74-0.92).

Conclusions: Significant risk factors for incident $A D$ were: tobacco smoking, household with visible moulds, older mother, traffic-related air pollution and high socioeconomic status. Long TV watching were associated with decreased risk of AD.

\section{PARENT-INITIATED ORAL PREDNISOLONE FOR EPISODES OF ACUTE ASTHMA IN CHILDREN AGED 5-13 YEARS}

\author{
P. Vuillermin ${ }^{1,2,3}$, C. Robertson ${ }^{2,3}$, J. Carlin $^{2,3}$, \\ S. Brennan ${ }^{1}$, M. Biscan ${ }^{1}$, M. South ${ }^{2,3}$ \\ ${ }^{1}$ Child Health Research Unit, Barwon Health, \\ Geelong, ${ }^{2}$ Murdoch Childrens Research Institute, \\ ${ }^{3}$ University of Melbourne, \\ Melbourne, VIC, Australia
}

Background: There is high-level evidence that oral corticosteroids are effective in the treatment of acute asthma in school-aged children when administered following physician review. However, existing data regarding the effectiveness of parent-initiated oral corticosteroids for acute asthma in children is both disappointing and inadequate. We therefore aimed to assess the efficacy of a short course of parentinitiated oral prednisolone for acute asthma in school-aged children.

Methods: We used a population-based sampling strategy to recruit children aged 5-12 years who had suffered four or more episodes of acute asthma in the preceding year. Episodes of acute asthma (rather than participants) were randomised to be treated with a 3-5 day course of parent-initiated prednisolone $(1 \mathrm{mg} / \mathrm{kg})$ or placebo. The primary outcomes were a 7-day daytime symptom score and health resource utilization (HRU).

Results: 230 children were enrolled in the study. Over a 3 year period 131 participants contributed 155 episodes of acute asthma randomised to parent-initiated treatment with prednisolone and 153 episodes treated with placebo. The mean daytime symptom score was on average 15\% (95\% Cl $2 \%$ to $26 \%, p=0.022$ ) lower among episodes treated with prednisolone. Concordantly, 48 (31\%) of episodes treated with prednisolone resulted in HRU compared with 69 (45\%) of episodes treated with placebo (odds ratio $=0.54,95 \% \mathrm{Cl} 0.34$ to 0.86, $p=0.010)$.

Conclusions: We found that a short course of oral prednisolone initiated by parents when their child suffers an episode of acute asthma resulted in a modest reduction in asthma symptoms and HRU. 